\title{
Consensus in Medicine
}

Caesarean childbirth

\author{
SUMMARY OF AN NIH CONSENSUS STATEMENT
}

A consensus development conference on caesarean childbirth was held at the National Institutes of Health on 22-24 September 1980. A consensus development task force and members of the audience considered evidence on the questions presented below. This summary is the result of their deliberations.

The rising caesarean birth rate is a matter of concern. The consensus statement reflects the judgment that this trend of rising caesarean birth rates may be stopped and perhaps reversed while maternal and fetal outcomes continue to improve -the goal of clinical obstetrics today.

(1) Why and how have caesarean delivery rates changed in the United States and elsewhere, and how have these changes affected pregnancy outcome?

Many factors have been cited to account for the increase in caesarean birth rates. In the 1960 s, when the overall birth rate was declining, increasing emphasis was placed on the health of the fetus. With couples having fewer children even greater attention was given to a favourable outcome of pregnancy. There were societal demands for preventing death of the fetus and neonate and for improving the quality of life for the survivors. The nation's infant mortality rate was used as an international yardstick of the quality of health care. Advances in medical care - such as improved anaesthetic techniques, blood products, and blood transfusions; wider choice of antibiotics for treating infection; and better medical control of maternal illnesses such as diabetes, hypertension, and heart disease-all made maternal mortality from caesarean childbirth rare. The safer the procedure became, the easier it became to decide to

This conference was sponsored by the National Institute of Child Health and Human Development, in conjunction with the National Center for Health Care Technology and with the help of the Office for Medical Applications of Research, NIH.

Members of the task force were: Dr Mortimer G Rosen (chairman), Case Western Reserve University, Cleveland, Ohio; Dr Milton H Alper, Harvard Medical School, Boston, Massachusetts; Dr Randall Bloomfield, Kings County Hospital Center Brooklyn, New York; Dr Robert C Cefalo, University of North Carolina Medical School, Chapel Hill, North Carolina; Dr Tiffany Field, University of Miami School of Medicine, Miami, Florida; Dr Jeanne Guillemin, Boston College, Chestnut Hill, Massachusetts; Dr Robert B Hilty, Kettering Medical Center, Dayton, Ohio; Dr Melita Jordan, Pennsylvania Hospital, Philadelphia, Pennsylvania; Dr Barbara F Katz, University of Massachusetts, Boston, Massachusetts; Dr Luella Klein, Emory University School of Medicine, Atlanta, Georgia; Dr Nicholas M Nelson, Pennsylvania State University, Hershey, Pennsylvania; Dr Diana Petitti, The Permanente Medical Group, Oakland, California; Dr Jack W Provonsha, Loma Linda University, Loma Linda, California; $\mathrm{Mr}$ Sam Shapiro, Johns Hopkins Medical Institutions, Baltimore, Maryland; Ms Elizabeth L Shearer, C/SEC, Inc, Chestnut Hill, Massachusetts; Dr Michael A Simmons, Johns Hopkins University School of Medicine, Baltimore, Maryland; Dr Judith Wagner, Urban Institute, Bethesda, Maryland; Dr S Annette Warrenfeltz, Waynesboro Hospital, Waynesboro, Pennsylvania; Dr Peggy J Whalley, University of Texas Southwestern Medical School, Dallas, Texas. perform the operation. Thus, caesarean delivery was one approach applied to try to improve fetal outcome.

Studies suggesting that caesarean delivery improved the outcomes of various complications of pregnancy led to its increased use for certain conditions. Obstetricians came to favour surgical relief of abnormal fetal presentations that formerly required a difficult forceps or other manipulative delivery. Dystocia was diagnosed more frequently and more often managed by caesarean delivery. Episodes of fetal distress during labour, more quickly detected by electronic fetal monitoring, were often managed by caesarean birth. As the primary caesarean section rate rose with more frequent surgical intervention for these complications, the long-held tenet: "Once a caesarean, always a caesarean" led to a rapid increase in the number of repeat caesarean births in subsequent pregnancies.

Some observers have suggested that the caesarean section rate has been affected by other factors, such as defensive medicine, financial incentives, and the trend towards specialisation in obstetric practice. Moreover, concern has been voiced about the degree to which patterns of residency training have perpetuated the trend. No reliable data exist to assess the extent to which any of these factors may have contributed to the rise in caesarean delivery rate.

\section{EPIDEMIOLOGICAL DATA}

During the 1970 s the caesarean birth rate in the United States increased about threefold, from $5.5 \%$ in 1970 to $15.2 \%$ in 1978 , and it appears to be continuing to increase. This trend is pervasive, affecting hospitals and patients in all parts of the country.

An increase in caesarean section rates is also evident internationally. The Canadian rate has most closely paralleled that of the United States, moving from $7.5 \%$ in $1972-3$ to $13.9 \%$ in 1979. In Western European nations, caesarean delivery rates are considerably lower than in Canada and the US, but a trend $\cong$ toward an increase in the proportions of caesarean delivery is reported in France, England, Norway, and The Netherlands. Other than Norway between 1975 and 1978, the US has experienced the sharpest increase.

Concurrent with the rise in caesarean delivery rates has been an international trend toward greater reliance on medical specialists for managing childbirth. This includes more deliveries performed by obstetricians than by general practitioners or midwives and better access to a full range of hospital resources.

In the United States the diagnostic categories having the largest effect on the increase in the caesarean birth rate between 1970 and 1978 are shown in the table. The experience of caesarean deliveries in New York City, an area for which data from birth and death certificates are available to assess changes in methods of delivery from 1968 to 1977 , is consistent with the national data. Along with the rise in the proportion of caesarean births, there has also been a trend away from forceps delivery. 
Diagnostic categories with largest effect on increase in caesarean delivery rates from 1970 to 1978

\begin{tabular}{|c|c|c|c|c|}
\hline Indication & & & $\begin{array}{c}\% \text { Of all caesarean } \\
\text { sections done for } \\
\text { this indication } \\
(1978)\end{array}$ & $\begin{array}{l}\% \text { Contribution } \\
\text { to rise in rate }\end{array}$ \\
\hline $\begin{array}{l}\text { Dystocia .. } \\
\text { Repeat caesarean section } \\
\text { Breech presentation } \\
\text { Fetal distress } \quad . .\end{array}$ & $\begin{array}{l}\ldots \\
\cdots \\
\cdots\end{array}$ & $\begin{array}{l}\cdots \\
\cdots \\
\cdots\end{array}$ & $\begin{array}{r}31 \\
31 \\
12 \\
5\end{array}$ & $\begin{array}{c}30 \\
25-30 \\
10-15 \\
10-15\end{array}$ \\
\hline
\end{tabular}

Low birth weight is associated with relatively high rates for primary caesarean birth. Because more than $90 \%$ of all infants weigh over $2500 \mathrm{~g}$ at birth, however, the group with normal birth weights makes the largest contribution to the number of caesarean births. The rate of increase in primary caesarean birth in New York City over the decade was similar at almost all birth weights. The incidence of low birth weight decreased both overall and among caesarean births. These changes in incidence of low birth weight run counter to the upward trend in caesarean delivery rates.

Neonatal mortality decreased sharply in New York City from 1967-8 to 1976-7. The largest decrease occurred in the group of infants weighing $1000-2500 \mathrm{~g}$. In this group there were large decreases in neonatal mortality after both primary caesarean deliveries and vaginal births. These decreases may have been influenced by concomitant improvements in factors such as neonatal intensive care. Among infants weighing over $2500 \mathrm{~g}$ there was a small decrease in neonatal mortality. No decrease was found in neonatal mortality among all primary caesarean births when shifts that may have occurred from forceps to caesarean delivery were allowed for.

(2) What is the evidence that caesarean delivery improves the outcome of various complications of pregnancy?

\section{DYSTOCIA}

The term dystocia encompasses two systems of classification of abnormal labour. The first relates to problems of fetal position or size. The second is less well-defined and includes functional classification of labour with a fetus in the vertex position. The largest contribution $(30 \%)$ to the overall rise in the caesarean delivery rate from 1970 to 1978 came from the diagnostic category of dystocia, mainly because of the increased frequency of the diagnosis of dystocia. In the New York City data the diagnosis of dystocia was concentrated among infants with birth weights over $2500 \mathrm{~g}$, and caesarean delivery was not associated with any advantage in survival over vaginal births. There are no adequate data on infant morbidity, and, specifically, abnormal neurological development, in association with dystocia.

Problems of fetal position and size (fetopelvic disproportion) are generally easily recognised, appropriate guidelines for obstetric management are available, and the effects on fetal and maternal outcomes are reasonably well known. These problems, excluding the breech birth, which is discussed as a separate category, are not a major reason for the increase in caesarean delivery rates among infants with dystocia.

The functional classification of labour as dystocia with a fetus in vertex presentation is more complex. The ability of the fetus weighing less than $1000 \mathrm{~g}$ to tolerate vaginal or caesarean delivery has not been adequately evaluated. Similarly, the fetus weighing more than $4000 \mathrm{~g}$, when recognised, requires particularly cautious obstetric management of labour. The remaining vertex births (over 90\%) are of normal size fetuses.

\section{Recommendations}

In the absence of fetal distress, management of dysfunctional labour may include such measures as rest, hydration, ambulation, sedation, and use of oxytocin before caesarean delivery is considered.

- There is a compelling reason to examine the diagnostic category of dystocia because of its prominent association with the increase in the primary caesarean birth rate and the absence of any advantage in survival for infants with birth weights over $2500 \mathrm{~g}$ delivered by caesarean section.

- Included among approaches to the issue of dystocia should be (a) peer review within hospitals; $(b)$ examination of the efficacy of methods for assessing the progress of labour, with specific attention to infant and maternal mortality and morbidity; and $(c)$ research clarifying the factors which affect the progress of labour, including the effects of emotional support, ambulation, rest, sedation, and oxytocin stimulation.

\section{REPEAT CAESAREAN DELIVERY}

Previous caesarean delivery is one of the two major reasons cited for caesarean delivery in second and subsequent pregnancies, and the number of caesarean sections performed for this reason is likely to increase further if present trends continue. This diagnostic category accounted for $25-30 \%$ of the increase in caesarean section rate from 1970 to 1978. After a caesarean delivery, more than $98 \%$ of women in the United States undergo a repeat caesarean delivery for any subsequent pregnancy. This practice began in the early 1900 s to avoid the risk of uterine rupture at the scar site during labour. At that time the "classical" caesarean incision (a vertical incision in the body of the uterus) predominated, and the caesarean birth rate was extremely low. The incidence of scar rupture is higher with the classical, low vertical, and inverted- $T$ incisions, than with the low-segment transverse incision. After an initial caesarean birth and during subsequent pregnancies and labour, the low-segment transverse uterine incision now in general use is associated with lower maternal and fetal morbidity and mortality. With any uterine scar, however, rupture is unpredictable.

A study of maternal and neonatal mortality rates shows that the practice of routine repeat caesarean birth is open to question. Repeat caesarean section carries twice the risk for maternal mortality of vaginal delivery, and maternal mortality after repeat caesarean section has not fallen since 1970 . At birth weights below $2500 \mathrm{~g}$ the New York City data showed a consistent disadvantage in terms of neonatal mortality for caesarean delivery compared with vaginal births; at birth weights above $2500 \mathrm{~g}$ caesarean deliveries had the same mortality as vaginal births. Data from national and international sources suggest that labour and vaginal delivery after a previous low-segment transverse caesarean birth is of low risk to mother and fetus in properly selected cases.

\section{Recommendations}

In hospitals with appropriate resources for prompt emergency caesarean delivery a proper selection of cases should permit a safe trial of labour and vaginal delivery for women who have had a previous low-segment transverse caesarean delivery. Informed consent should be obtained before a trial of labour is attempted.

- No changes in present practices are recommended for the delivery by elective repeat caesarean section of patients who have had a previous classical, inverted-T, or low vertical incision, or for whom there is no information on the site and type of the previous caesarean incision.

- In hospitals without appropriate resources the risk of a trial of labour in women who have had a previous caesarean delivery may exceed the risk for both mother and infant from a properly timed, elective repeat caesarean birth. Patients should be told in advance of the limits of a particular hospital's capabilities and of the availability of other hospitals capable of offering this service, so that they may make a choice. 
- More adequate information is needed on risks and benefits in trials of labour for patients with previous low-segment transverse uterine incisions. Studies designed to provide this information must have a large enough sample and include information on morbidity and mortality in both mother and infant.

- Hospitals offering trials of labour after a low-segment transverse caesarean birth should develop guidelines for the management of these deliveries.

- Education of the patient about caesarean birth and repeat caesarean birth should continue throughout pregnancy so that she can participate in decisions about anaesthesia, elective repeat caesarean birth, or trial of labour after a previous caesarean birth.

\section{BREECH PRESENTATION}

Breech presentation is associated with an increase in both morbidity and mortality when compared with vertex presentation, irrespective of whether delivery is vaginal or caesarean. There is a continuing trend towards delivery of infants in breech presentation by caesarean section. Nationally the proportion of breech presentations delivered by caesarean section rose from $11.6 \%$ in 1970 to $60.1 \%$ in 1978, accounting for about 10 to $15 \%$ of the rise in caesarean section rate during those years.

Among breech presentations in the New York City data there was no consistent difference in neonatal mortality at low birth weights ( $2500 \mathrm{~g}$ or less) between primary caesarean births and vaginal breech deliveries. But at mature birth weights breechpresenting infants delivered by caesarean section had a fivefold better neonatal mortality than those delivered vaginally. The strong qualification, however, is that at birth weights above $2500 \mathrm{~g}$ there has been no overall decrease in mortality over 10 years for the total group of breech presentations (vaginal and caesarean combined). This seeming contradiction may reflect a shift of the better-risk breech births from the vaginal delivery group to the caesarean birth category. Further exploration of the effect of changes in mode of delivery would require information on infant morbidity and development, which is not available.

Evaluation of outcome in breech presentation is complicated by the increased frequency of prematurity in breech presentations, the different types of breech presentation, congenital anomalies associated with breech presentation, and the influence of maternal pelvic size and fetal size on ease of vaginal breech delivery. Maternal morbidity after surgery or difficult breech delivery is a consideration in choosing the method of delivery. Most clinical reviews suggest that abdominal breech delivery may be associated with less risk to the premature fetus, but lack of data on infant morbidity prevents a firm conclusion.

The large fetus presenting as breech, the fetus presenting as a complete or footling breech, and the fetus with pronounced hyperextension of the head presenting as breech all have a better outcome if delivered by caesarean birth.

\section{Recommendations}

Vaginal delivery of the term breech should remain an acceptable obstetrical choice for delivery when the following conditions are present:

(a) anticipated fetal weight of less than $3630 \mathrm{~g}$;

(b) normal pelvic dimensions and architecture;

(c) frank breech presentation without a hyperextended head;

(d) delivery to be conducted by a physician experienced in vaginal breech delivery.

- There are too few data to make a firm recommendation on the preferable method of delivery for the low birthweight (less than $2500 \mathrm{~g}$ ) fetus in the breech presentation.

- More data should be collected to evaluate the best mode of delivery for fetuses in the breech presentation.
Because all breech births have inherent risks that are often uncertain and unpredictable, this information should be shared with the family whenever possible as part of the decision-making process.

\section{FETAL DISTRESS}

Asphyxia during labour, which may lead to brain damage, is the most dangerous clinical component of fetal distress. Fetal distress ranks third in importance, along with breech presentation, in the rise of caesarean birth rates, accounting for about 10 to $15 \%$ of the increase. While evidence is lacking that the actual incidence of fetal distress has changed, the diagnosis of fetal distress has been made more often during the past 10 years. The use of electronic fetal monitoring is associated with an increased incidence of the diagnosis, and fetal distress is diagnosed most often in fetuses weighing over $2500 \mathrm{~g}$. Fetal distress cannot be reliably explored within the New York City data for risk of mortality in relation to method of delivery because the actual number of affected infants was small.

\section{Recommendation}

Further studies are needed to improve the accuracy of the diagnosis of fetal distress, and to develop new techniques for making the diagnosis. These advances may be expected to improve fetal outcome and lower caesarean birth rates.

\section{OTHER MATERNAL AND FETAL CONSIDERATIONS}

Because of a need for early delivery, many maternal and fetal medical problems lead to caesarean birth. Examples include maternal diabetes, pregnancy-induced hypertension, and erythroblastosis fetalis. This entire group has contributed only a small part of the increase in caesarean birth rate.

\section{Recommendation}

- If vaginal delivery is to be used in this group an effective method is needed to stimulate preterm labour safely.

(3) What are the medical and psychological effects of caesarean delivery on the mother, infant, and family?

\section{MATERNAL MORTALITY AND MORBIDITY}

Although overall maternal mortality is extremely low (9.9 deaths/100 000 births in 1978), caesarean birth carries two to four times the risk of mortality when compared with vaginal delivery. Some maternal deaths after caesarean birth are related to maternal illness rather than to the surgery. Maternal mortality is still underreported.

Caesarean birth is a major surgical procedure with morbidity greater than that of vaginal delivery. Infections constitute the greatest portion of this morbidity; the most common are endometritis and urinary tract and wound infections. Morbidity may reflect the demographic features of the population cared for as well as the events of labour and birth.

\section{Recommendations}

Better data collection is needed to identify the reasons for operative and non-operative maternal deaths.

- The National Center for Health Statistics should consider revising (a) the standard certificates of birth and fetal death to include items that distinguish between caesarean and vaginal delivery, and $(b)$ the standard certificates of death to include or 
modify items that improve the identification of maternal deaths. State health departments should adopt similar revisions in certificates of birth and death and a uniform definition of maternal death.

- For statistical analysis maternal death certificates should be matched with the corresponding birth and fetal death certificates.

- Comprehensive studies of morbidity with respect to caesarean births, vaginal births, and forceps births are needed.

- Delivery of medical care to patients undergoing caesarean birth should be made equal to that available to general surgical patients.

\section{ANAESTHESIA}

Obstetric anaesthesia is unique in that it requires attention to the health of at least two patients. Maternal deaths related to anaesthesia, although infrequent, continue to occur. Most anaesthesia-related deaths are potentially avoidable. Appropriate anaesthetic selection and technique can minimise the inherent medical risks, including the risk of maternal death. There is considerable variation and room for improvement in the number, quality, and availability of obstetric anaesthesia services throughout the United States.

\section{Recommendations}

- Choices about the kind of anaesthesia should be available and discussed among patient, obstetrician, and anaesthetist. In particular, the patient should have the option of receiving regional anaesthesia, when this is not contraindicated medically. - More comprehensive and continuing data collection is needed to determine the training, availability, and quality of anaesthetic support systems and staff for the obstetric patient. - Further study of the effects of obstetric anaesthesia on maternal mortality and morbidity and the physiology and conduct of labour is required.

- The short- and long-term effects of anaesthetic drugs and techniques on the neurobehavioural development of the newborn are poorly described in existing reports. Understanding these effects is critical to an evaluation of benefits and risks to infant development, and they warrant further study.

\section{NEONATAL RESPIRATORY DISTRESS SYNDROME}

Caesarean birth, particularly in the absence of labour, appears to be associated with an increase in neonatal respiratory distress at all gestational ages. Iatrogenic neonatal respiratory distress due to premature termination of pregnancy should be avoidable by appropriate antenatal use of clinical, biophysical, and biochemical estimators of gestational age. Among fetuses with lungs previously shown to be mature, postnatal respiratory distress is unlikely to be a problem, whatever the route chosen for their delivery.

\section{Recommendations}

- Continuing efforts to avoid iatrogenic prematurity are needed. There must be improvement in professional and patient education regarding the necessity for safe and effective antenatal assessment of gestational age and fetal maturity before elective intervention in labour and delivery.

- Further studies are needed of the influence on neonatal pulmonary function of events surrounding labour and delivery.

\section{PSYCHOLOGICAL AND DEVELOPMENTAL EFFECTS}

There is limited research concerning the psychological impact on parents of a caesarean birth. Nevertheless, surgery is clearly an increased psychological and physical burden when compared with a normal vaginal delivery. Negative responses from mother and father have also been reported in the available retrospective studies. In some hospitals family-centered maternity care has been extended to families where the mother has undergone caesarean delivery, and in these cases there is no evidence of harm to mother, neonate, or father. The presence of fathers in the operating room and closer contact between mother and neonate appear to improve the behavioural responses of the families after caesarean delivery. One consistent finding from small scale studies of these families has been the greater involvement of fathers with their infants. Improved educational programmes for all families so that they may understand the caesarean birth, and specific educational programmes for those who have already experienced a caesarean birth are methods to improve the birth experience.

Comparative studies of the influence of caesarean birth on infant development often lack a statement on the obstetric indications for caesarean delivery or whether the father was present in the delivery room. Several studies have noted that, although the infant delivered by caesarean section has usually received more medication than a control infant delivered vaginally, assessments of neonatal behaviour did not reveal depressive effects. Virtually no differences associated with mode of delivery have been found in the few reports available on infant development beyond the neonatal period. The published studies are often inadequate in design and incomplete in their follow-up of the infants.

\section{Recommendations}

- Parent education during pregnancy by health-care providers and in childbirth education classes should include information on the possibility of a caesarean birth, an explanation of the technical procedures, and discussion of choices available to parents.

- During labour and at the time a decision to perform a caesarean delivery is made, as time and circumstances permit, a discussion of the indications, procedures, and options should take place between the doctor or his or her staff and the parents. - Education about the entire caesarean birth experience should continue after the operation and at later postpartum visits.

- In the absence of scientific evidence of benefit or risk, the presence at a caesarean birth of the father or surrogate should be the result of a joint decision of parents, doctor, and hospital representatives.

- Hospitals are encouraged to liberalise their policies on having the father or surrogate attend the caesarean birth.

- The healthy neonate should not be separated routinely from mother and father after delivery.

- There is a need for longitudinal developmental follow-up studies of infants and their parents. These studies should include study populations of sufficient size for meaningful statistical analysis, suitable control groups, and standardised methods of assessment.

\section{(4) What economic factors are associated with caesarean} births?

The medical cost of delivery by caesarean birth is generally higher than that of vaginal delivery. The available evidence does not permit a precise assessment of the value of health care resources that would be saved by avoiding a caesarean birth. These additional medical costs can be assessed only in relation to their benefits to both infant and mother. At present information on benefits, particularly those relating to morbidity and development of the infant, is inadequate to allow their comparison with cost.

Historically, there have been financial incentives to patients both for and against caesarean delivery. In women without 
health insurance an additional financial burden is imposed by the caesarean birth. Conversely, many employer-sponsored health insurance plans, where coverage of normal deliveries has been limited, have given financial advantages to the caesarean birth. This latter incentive is disappearing owing to a change in civil rights legislation in 1979. Evidence on the effect of these incentives on caesarean birth rates is insufficient.

Financial incentives to doctors both for and against the performance of caesarean birth may exist. In some cases the higher fee paid for caesarean delivery may not be justified by greater effort or time. In other cases-for example, in the case of family practitioners who must refer patients to a specialist for caesarean delivery-incentives against a caesarean birth may exist. Evidence on the effects of incentives for doctors on caesarean birth rates is limited and conflicting at present.

\section{Recommendations}

- Although it would be useful to know how the medical benefits of caesarean birth compare with its costs in various situations, no recommendation can be made to conduct economic evaluations on this issue until these benefits can be better defined and measured.

- Research is needed into the economic incentives for and against caesarean birth and into the effect of these incentives on the behaviour of doctors and patients.

(5) What legal and ethical considerations are involved in decisions on caesarean delivery?

LEGAL CONCERNS

Malpractice suits may be brought against a doctor for negligent performance of a caesarean section, for not performing a necessary caesarean operation, or for performing an unnecessary one. Of major concern is the malpractice suit for negligent nonperformance of an allegedly necessary caesarean. The doctordefendant is successful in most of these cases. Liability, when found, generally involves gross deviation from the recognised standards of patient care. Appropriate application of the doctrine of informed consent by providing a patient with sufficient information to make a knowledgeable choice of treatment protects the doctor. The law also protects the rights of the unborn child. Although commonly cited, there is no evidence that fear of litigation and the possible consequent practice of defensive medicine is a major cause of the increased caesarean birth rate.

\section{Recommendations}

- The courts should recognise that if a vaginal delivery resulted in a "less than perfect" baby, this does not necessarily mean that the doctor was negligent for not performing a caesarean birth. - Doctors should make a determination about the need for a caesarean delivery based solely on sound medical judgment.

- Doctors should support the patient's right to participate in the decision whether to have a caesarean delivery by proper application of the doctrine of informed consent.

\section{ETHICAL CONCERNS}

The ethical principles associated with caesarean birth are generally not specific to this procedure. They correspond to established patterns governing relationships between health-care providers and their patients. They are consistent with providers' traditional commitment to giving patients' interests priority over their own; they acknowledge the right of patients to make informed decisions regarding their own bodies, including the right of proxy consent; they recognise the independent rights of the unborn relative to those of the mother; and they take into account the special needs and concerns of teaching institutions and the ethical difficulties involved in achieving an equitable distribution of health care.

\section{Recommendation}

- Further studies are needed to discover whether the increase $\stackrel{D}{\mathbb{D}}$ in caesarean delivery rates in fact represents a violation of any $m$ of these general principles.

\section{Research needs}

Throughout this consensus statement recommendations $\frac{\bar{\sigma}}{\frac{\sigma}{\sigma}}$ appear for the development of new data and research studies. $\triangle$ The task force has found at all stages of its assessment of $\approx$ caesarean childbirth that evidence to reach hard conclusions is $\vec{\circ}$ often not available. In part this may be due to the complexity of the subject; in part it may be due to the lag in developing $\vec{\omega}$ information that identifies the critical questions needing $\frac{\partial}{\partial}$ intensive investigations. The epidemiological analysis of the $\frac{3}{3}$ national and New York City experiences has clarified many of these issues. There is a need for data similar to New York City's for other population groups. Nevertheless, studies that have the potential for more effectively answering questions concerning the reasons for and efficacy of caesarean birth need to be conducted urgently. This will require a range of different methods, 8 including well-designed record-based sources of information, interview surveys, case control studies, and randomised clinical $\stackrel{5}{\supset}$ trials.

Randomised clinical trials are difficult, time consuming, and expensive. In certain circumstances, however, they offer the only way of addressing problems definitively. The question of $\vec{\omega}$

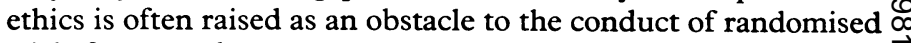
trials for procedures or managements which are already routine, whether or not they are established. Failure to conduct feasible randomised trials in the case of clinical practices whose efficacy is not established and is being persuasively challenged, however, raises ethical issues of greater concern.

Another area that needs research is doctors' practices. The $\stackrel{\mathbb{Q}}{\varrho}$ effects of medical training, hospital policy, and ethical and legal $\overrightarrow{\vec{O}}$ concerns on the choice of delivery procedure are currently 3 unexamined subjects deserving greater scrutiny by means of a $\bar{F}$ combination of epidemiological, economic, and social science methods.

Most major issues concerning caesarean birth will require longitudinal evaluation of infants. Such studies are expensive and logistically difficult, but they should be regarded as high priority areas by funding agencies.

Copies of the full report of the task force, containing a description and analysis of the literature and data on which this statement is based, may be obtained from the Office of Research Reporting, $N$ NICHD, Building 31, Room 2A34, NIH, 9000 Rockville Pike, > Bethesda, Maryland 20205. Is there any reliable evidence that sexual intercourse during menstruation
is harmful?

There is no medical contraindication to intercourse during menstrua- 0 tion. Most couples refrain from intercourse while the woman is $\overrightarrow{0}$ bleeding, though by no means all do so. ${ }^{1}$ The tendency to abstain is $\overrightarrow{\mathbb{D}}$ partly explained by decreased libido, though this shows much indi- $\frac{\rho}{\mathbb{D}}$ vidual variation. Abstinence may also owe something to religious or primitive taboos, but it probably has more to do with practical considerations, such as laundry bills.

${ }^{1}$ Udry JR, Morris NM. The distribution of events in the human menstrual cycle.
$\mathcal{F}$ Reprod Fert $1977 ; 51: 419-25$. 